\title{
Fluticasone furoate maintains epithelial homeostasis via leptin/ leptin receptor pathway in nasal cells
}

\author{
Andreina Bruno - Stefania Gerbino • Maria Ferraro • Liboria Siena • \\ Angela Bonura • Paolo Colombo • Stefania La Grutta • Salvatore Gallina • \\ Antonella Ballacchino - Marco Giammanco • Mark Gjomarkaj • Elisabetta Pace
}

Received: 16 January 2014/ Accepted: 11 July 2014

(C) Springer Science+Business Media New York 2014

\begin{abstract}
Leptin is involved in the lung epithelial homeostasis. Its role in the nasal tract is largely unknown. Allergic rhinitis (AR) is induced by the allergen exposure leading to consequential structural abnormalities in the nasal epithelium. Topical corticosteroids are recommended as first-line therapy in AR. Parietaria pollen is one of the most important allergenic sources in the southern Europe. In vitro, in human nasal epithelial cell line RPMI 2650, we aimed to determine whether allergen stimulation acts on leptin/leptin receptor pathway and how fluticasone furoate (FF) influences this pathway. The effects of the major allergen recombinant Par $\mathrm{j} 1$ (rPar j 1), of FF, of leptin, and of TGF- $\beta 1$ on cell proliferation, on leptin/leptin receptor expression and modulation (by clonogenic test, by RT-qRT-PCR, by immunocytochemistry and by flow-cytometry), and on STAT-3 activation (assessing nuclear
\end{abstract}

Electronic supplementary material The online version of this article (doi:10.1007/s11010-014-2142-z) contains supplementary material, which is available to authorized users.

A. Bruno $(\square) \cdot$ S. Gerbino - M. Ferraro - L. Siena - A. Bonura .

P. Colombo · S. La Grutta $\cdot$ S. Gallina · A. Ballacchino .

M. Gjomarkaj · E. Pace ( $\square)$

Consiglio Nazionale delle Ricerche (CNR), Institute of

Biomedicine and Molecular Immunology (IBIM), Via Ugo La

Malfa, 153, 90146 Palermo, Italy

e-mail: andreinabrn@gmail.com

E. Pace

e-mail: pace@ibim.cnr.it

S. Gerbino · M. Giammanco

Unit of Physiology and Pharmacology, Department of Sport

Science, University of Palermo, Palermo, Italy

S. Gallina

Sezione di Otorinolaringoiatria, Dipartimento Biotecnologie e

Neuroscienze Cliniche, University of Palermo, Palermo, Italy translocation by western blot analysis) were assessed. We found that $r$ Par $\mathrm{j} 1$ and TGF- $\beta 1$ significantly decreased cell proliferation and down-regulated the leptin/leptin receptor pathway, whereas FF and leptin reverted them, both alone and in combination. Furthermore, rPar j 1 reduced, while leptin and FF increased STAT-3 activation. In conclusion, $\mathrm{FF}$ and leptin itself are able to preserve nasal epithelial homeostasis restoring the leptin/leptin receptor pathway altered by rPar j 1 exposure.

Keywords Allergic rhinitis - Epithelium - Fluticasone furoate $\cdot$ Leptin $\cdot$ rPar j 1
Abbreviations
AR Allergic rhinitis
FF Fluticasone furoate
rPar j 1 Recombinant Par j 1

\section{Introduction}

Leptin adipokine bridges the nutritional status and the immune system, as it has been recognized to be a pleiotropic molecule involved in a variety of physiological functions in both the central nervous system and the periphery [1,2]. Leptin exerts its roles through several forms of its receptor, expressed in numerous cytotypes including neurons, epithelial cells, and immune cells. Upon leptin binding to the extracellular binding domain of its receptor long form, ObRb, JAK2 becomes activated and phosphorylates three tyrosine residues on ObRb (Tyr 985, 1077, and 1138) that are in turn recognized by STAT3. Following phosphorylation of STAT3, there is initiation of 
multiple downstream signaling events mediates by leptin [3]. Long-term leptin replacement therapy restores CD4 numbers, IFN- $\gamma$ expression, and T-cell function while decreases TGF- $\beta$ to normal levels [3]. In the lung, recent observations suggest a modulatory role for leptin in pulmonary development and identify the leptin receptor as a physiological marker of fetal lung maturity [2] and of epithelial cell proliferation [4]. In the respiratory tract, leptin has a role in the regulation of immune cells $[2,5]$ as well as in maintaining epithelial homeostasis [6]. The positive regulation of cell proliferation is an event crucially involved in the repair processes also in nasal epithelium. In the nasal tract, upon injury, undifferentiated basal cells migrate from adjacent epithelium, spread over the denuded basement membrane, and proliferate in injured regions [7]. Despite leptin playing different roles in the olfactory mucosa cells [8], in the human nasal epithelium, its role is still largely unexplored.

Allergic rhinitis (AR) affects 400 million people worldwide [9]. It is a chronic inflammatory disorder mediated by response to natural allergen exposure and represents an important health problem due its prevalence and its impact on patients' social life, school-performance, and work productivity [10]. Often AR is part of a systemic inflammatory process associated with other inflammatory disorders including asthma. A previous our in vitro and ex vivo study aimed to assess the role of leptin/leptin receptor on asthma remodeling concludes that this pathway is involved in lung epithelial homeostasis in asthma, and its expression is inversely correlated with airway remodeling, including reticular basement membrane and TGF- $\beta 1$ expression [6].

The RPMI 2650 nasal epithelial cell line represents a valid in vitro model to evaluate the regulation of TGF- $\beta$ biology [11] and for rhinoshinutis [12]. The uses of intranasal corticosteroids, patients' education, and prevention of allergen contact are all important tools for optimal symptom control in AR patients [9]. Particularly, the treatment with intranasal fluticasone propionate for 1 year increases the thickness of the nasal epithelium, and this phenomenon could be associated to repair events in response to injury due to chronic inflammation [13]. Parietaria judaica belongs to the genus of dicotyledonous weeds of the $U r$ ticaceae family, and its pollen grain is one of the major sources of allergens in the Mediterranean area. Its major allergens (Par j 1 and Par j 2) have been sequenced and characterized [14, 15]; particularly, recombinant Par $\mathrm{j} 1$ ( $r$ Par j 1) is a small protein of $14.400 \mathrm{Da}$, belonging to "Plant non-specific lipid transfer proteins" (nsLTP), small cysteine-rich lipid-binding proteins involved in plant resistance to biotic and abiotic stresses, widely distributed in the plant kingdom. Based on all these evidences, the present study was performed to investigate, in vitro in
RPMI cells, the role of leptin/leptin receptor pathway on epithelial homeostasis in the presence or absence of fluticasone furoate (FF), of rPar j 1, of leptin itself, and of TGF$\beta 1$.

\section{Materials and methods}

\section{Reagents}

rPar j 1, fully named Par j 1.0201 (EMBL accession number X85012), was generated as previously described [14]. This recombinant protein was tested for the endotoxin content using the multi-test limulus amebocyte lysate (LAL) pyrogen plus test (Bio-Whittaker, USA) (endogenous endotoxin content $\leq 0.003 \mathrm{ng} \mathrm{LPS} / \mu \mathrm{g}$ of recombinant protein). FF was provided by GlaxoSmithKline, and it was solved in $\mathrm{N}, \mathrm{N}$-dimethylacetamide (D5511) was purchased from Sigma Aldrich. Recombinant human leptin and recombinant human TGF- $\beta 1$ were purchased from R\&D Systems. The polyclonal anti-leptin (Ob A-20: sc 842), anti-leptin receptors (Ob-R M-18: sc1834), the monoclonal anti-STAT-3 (STAT-3 F-2: sc8019) antibodies, and the fat dry milk were from Santa Cruz Biotechnology. Pharmacological JAK2 inhibitor AG 490 (cat\# 658401) was from Calbiochem. TRIzol Reagent and oligo(dT)12-18 primer were from Invitrogen, FAM-labeled probe, and primers from Applied Biosystems.

\section{RPMI 2650 cultures and treatments}

RPMI 2650 cell lines (ATCC-CCL-30) were purchased from American Type Culture Collection (ATCC; Rockville, MD, USA). Cells were cultured in complete culture medium (MEM minimum essential media containing $10 \%$ FCS, L-glutamin $2 \mathrm{mM}$, gentamicin $50 \mathrm{mg} / \mathrm{ml}$, MEM NEAA $0.5 \%$, sodium pyruvate $1 \mathrm{nM}$, HEPES $1 \%)$ in the presence or absence of $\mathrm{rPar} \mathrm{j} 1(1 \mu \mathrm{g} / \mathrm{ml})$, FF $\left(10^{-8} \mathrm{M}\right)$, recombinant human leptin $(0.5 \mu \mathrm{M})$ [6], and recombinant human TGF- $\beta 1$ ( $2 \mathrm{ng} / \mathrm{ml})$ [6], alone or together combined, for $24 \mathrm{~h}$. Preliminary experiments for leptin receptor expression were performed to establish the best drug concentration (Fig. S1) and the best time point (Fig. S2).

Stainings for leptin and leptin receptors

Cytospins were prepared from RPMI 2650 cultured without any stimulations, and cells were fixed and permeabilized as previously described [6]. After washing in PBS, slides were incubated with the primary antibodies anti-leptin $(1: 40,1 \mathrm{~h}$ RT) and anti-leptin receptor against the common part of the 
short and long isoform $\left(1: 50\right.$, overnight $\left.4{ }^{\circ} \mathrm{C}\right)$. BSA $0.5 \%$ in TBS was used as blocking agent.

The reaction was revealed by universal LSAB KIT phosphatase method (DAKO Glostrup, Denmark) including a mixture of secondary antibodies at fixed concentration. Non-immune $\operatorname{IgG}$ at the same titer as the primary antibodies was used as a negative controls. Cytospins were examined under light microscopy with a final magnification of $1,000 \times$. Moreover, to better gain inside to the mechanism of allergen exposure, immunohistochemistry stainings were also performed as previously described [6] on 5- $\mu \mathrm{m}$-thick paraformaldehyde-fixed paraffin-embedded tissue sections from inferior turbinate of nasal biopsies from three healthy not allergic control subjects and from three allergic patients. The study protocol was approved by the Policlinico Ethic Committee (13/2013) and informed written consent was obtained from each patient. Immunostainings were performed at $\mathrm{RT}, 1 \mathrm{~h}$ using rabbit polyclonal anti-leptin $(1: 40)$ and at $4{ }^{\circ} \mathrm{C}$ overnight using goat polyclonal anti-leptin receptor (1:50). Control Abs were used as negative controls. Slides were analyzed by Leica light microscope $(1,000 \times)$ by using Quantimet $500 \mathrm{MC}$ software (Leica) for image analysis.

Flow-cytometry for leptin and leptin receptor proteins

RPMI 2650 cell line was analyzed for leptin/leptin receptor expression by FACScalibur (Becton-Dickinson, Mountain View, CA, USA) flow cytometer in the presence of the described stimuli. FF diluent ( $N, N$-dimethylacetamide D5511), at the same concentration used to dilute FF, was also tested. Detection of intracellular leptin and of leptin receptors was performed as previously described [4] by using the specific polyclonal antibodies for leptin and for leptin receptors. To evaluate the expression of intracellular leptin, cells were treated overnight with GolgiStop $(2 \mu \mathrm{M}$ final concentration) (BD PharMingen) and fixed with PBS containing $4 \%$ paraformaldehyde for $20 \mathrm{~min}$ at room temperature. Fixed cells were washed twice in permeabilization buffer (PBS containing $1 \%$ FBS, $0.3 \%$ saponin, and $0.1 \% \mathrm{Na}$ azide) for $5 \mathrm{~min}$ at $4{ }^{\circ} \mathrm{C}$ and then incubated with rabbit polyclonal antibody anti-leptin (1:25; for $1 \mathrm{~h})$. For assessing leptin receptors, unpermeabilized cells were incubated with goat polyclonal anti-leptin receptor antibody $(1: 25$; for $1 \mathrm{~h})$. Polyclonal Swine Anti-Rabbit Immunoglobulins/FITC (Dako; Code Nr. F 0054) (1:25; for $1 \mathrm{~h})$ for leptin and polyclonal Rabbit Anti-Goat Immunoglobulins/FITC (Dako; Code Nr. F 0250) (1:25; for 1 h) for leptin receptor were used as secondary antibodies. Percentages of positive cells for leptin receptors and mean of positive cells for leptin were determined from forward (FS) and sideways (SS) scatter patterns, after gating on the cells, excluding debris. Non-specific binding and background fluorescence were quantified by analyzing negative controls.

\section{Clonogenic assay}

The colony growth of RPMI 2650 cells exposed to the previously described stimuli was assessed. In addition, the condition with pharmacological JAK2 inhibitor AG 490, alone or in combination with FF, with leptin, and with $r$ PRJ1 was performed. Using 35-mm Petri dishes (Falcon, Becton-Dickinson, Franklin Lakes, NJ, USA), a lower layer was prepared using complete MEM medium in $0.5 \%$ agarose. The cells were harvested and seeded $\left(5 \times 10^{4}\right)$ on the upper layer with $0.3 \%$ agarose prepared with the same medium as the lower layer and finally incubated for 21 days at $37{ }^{\circ} \mathrm{C}$ in an atmosphere containing $5 \% \mathrm{CO}_{2}$. At the end of incubation, colonies were counted under an inverted phase-contrast microscope (Leitz, Wetzlar, Germany). The experiments were conducted in triplicate. Colonies were defined as cell aggregates with at least 40 cells. The true number of colonies was calculated as the number of aggregates on the positive control subtracted from the number of colonies on the experimental plates. Results are expressed as percentages of the control value.

Quantitative real-time reverse transcription-polymerase chain reaction (RT-PCR) of leptin receptors

Total RNA was extracted from RPMI 2650 cells and cultured with TRIzol Reagent (Invitrogen, Carlsbad, CA, USA) in the presence of the previously described stimuli, following the manufacturer's instructions. $4 \mu \mathrm{g}$ of total extracted cellular RNA was reverse-transcribed, using M-MLV-RT and oligo(dT)12-18 primer (Invitrogen), into cDNA in a $20 \mu \mathrm{l}$ volume reaction mixture. Furthermore, $10 \% \mathrm{cDNA}$ aliquots $(2 \mu \mathrm{l})$ from reverse reaction were used as templates for realtime PCR amplification (approximately $20 \mathrm{ng}$ of cDNA). Quantitative real-time PCR of leptin and of the common part of the short and long form of human leptin receptor transcripts was carried out on Step One Plus Real-time PCR System (Applied Biosystems, Foster City, CA, USA) using specific FAM-labeled probe and primers (prevalidated TaqMan Gene expression assay for leptin, Hs00174877m1, and for leptin receptor, Hs00174492m1; Assays on Demand, Applied Biosystems). Leptin and leptin receptor gene expression was normalized to glyceraldehyde-3-phosphate dehydrogenase (GAPDH) endogenous control gene. Relative quantization of gene expression was calculated with the comparative CT method $\left(2^{-\Delta \Delta C_{\mathrm{T}}}\right)$ [15] and was plotted as fold-change compared to untreated cells, chosen as the reference sample. 
Fig. 1 RPMI 2650 cells express leptin and leptin receptors.

a Immunocytochemistry was performed for assessing leptin and leptin receptor in RPMI 2650 cells. Right corners negative controls. Magnification at $\times 1,000$. b Flow-cytometry analysis was performed for assessing leptin and leptin receptor in RPMI 2650 cells. Overlay of fluorescence intensity (FL1) related to expression of leptin and leptin receptors versus events histogram of cells cultured in medium. Left gray peaks negative controls
Leptin expression
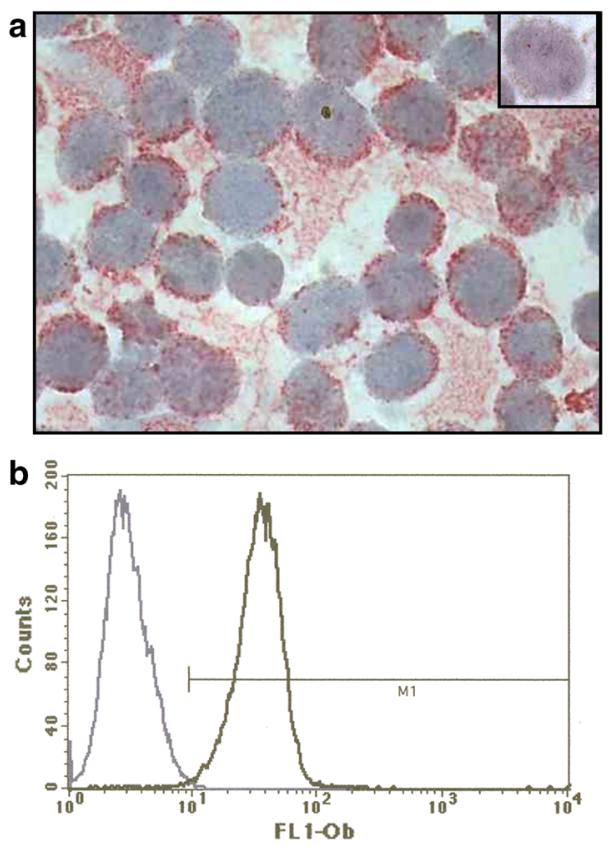

Leptin Receptor expression
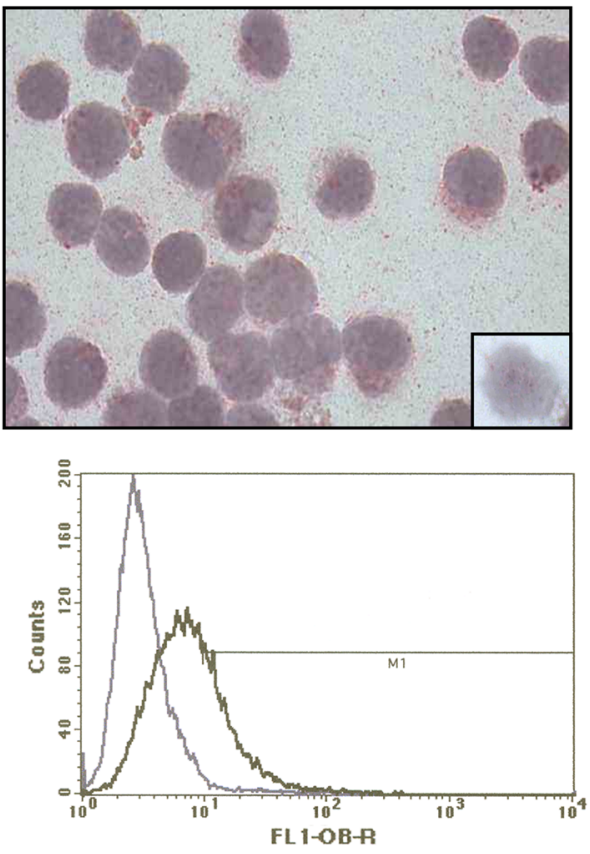

Western blot analysis for STAT-3

Western blot analysis for Signal transducer and activator of transcription (STAT)-3 was performed as previously described [16] with some modifications. To study STAT-3 nuclear translocation, the protein extracts were treated to separate the cytoplasmic and nuclear protein fractions by using a commercial kit "NE-PER Nuclear and Cytoplasmic Extraction Reagents" following the manufacturer's directions (Thermo Scientific; Waltham, MA, USA). An amount of $40 \mu \mathrm{g}$ of total proteins was subjected to sodium dodecyl sulfate-polyacrylamide gel electrophoresis (SDS-PAGE) on $10 \%$ gels and blotted onto nitrocellulose membranes. These were blocked with PBS containing $5 \%$ no fat dry milk (for $1 \mathrm{~h}$; room temperature), $0.1 \%$ Tween 20 , and then probed with the antibody anti-Stat 3 (1:100; overnight) and a goat anti-mouse antibody $(1: 1,000 ; 1 \mathrm{~h})$ (Amersham Biosciences, Piscataway, NJ, USA). Revelation was performed with an enhanced chemioluminescence system (GE Healthcare, Chalfont St. Giles, UK) followed by autoradiography. The expression of beta-actin (Sigma) and lamin B1 as housekeeping proteins for cytoplasmic and nuclear extracts, respectively, was assessed by using goat polyclonal anti- $\beta$-actin and rabbit polyclonal anti-lamin B1 (Cell Signaling, Danvers, MA, USA).

\section{Statistical analysis}

Results are expressed as mean $\pm \mathrm{SD}$. Paired $t$ test was performed. Kolmorogov-Smirnov Normality test was initially performed to assess whether parametric analyses of data could have been performed. A P value of less than 0.05 was considered as statistically significant.

\section{Results}

RPMI 2650 cells express leptin and its receptors

In our in vitro model, we first assessed, by immunocytochemistry analysis and by flow cytometry, whether RPMI 2650 cells constitutively expressed leptin and its receptor proteins. These cells expressed both proteins in the absence of any stimuli (Fig. 1a, b). Ex vivo approaches confirmed that nasal epithelial cells expressed leptin and its receptors (as demonstrated by a red ring staining) and showed a trend of reduction for leptin and leptin receptor expressions in allergic patients in comparison to control subjects (Fig. 2a, b). Negative control Abs demonstrated no significant immunostainings (data not shown).

Effect of rPar j 1, FF, and cytokines on RPMI 2650 cell proliferation

Since leptin/leptin receptor pathway exerts a proliferative role in various cell lines [17] including some bronchial epithelial cell lines $[4,6]$, we investigated whether this pathway was involved in the proliferative events (assessed by colony numbers) of nasal epithelial cells and whether rPar $\mathrm{j} 1$, leptin, and TGF- $\beta 1$, in the absence and in the presence of FF. Interestingly, significantly higher colony numbers were observed in RPMI 2650 cells cultured with 

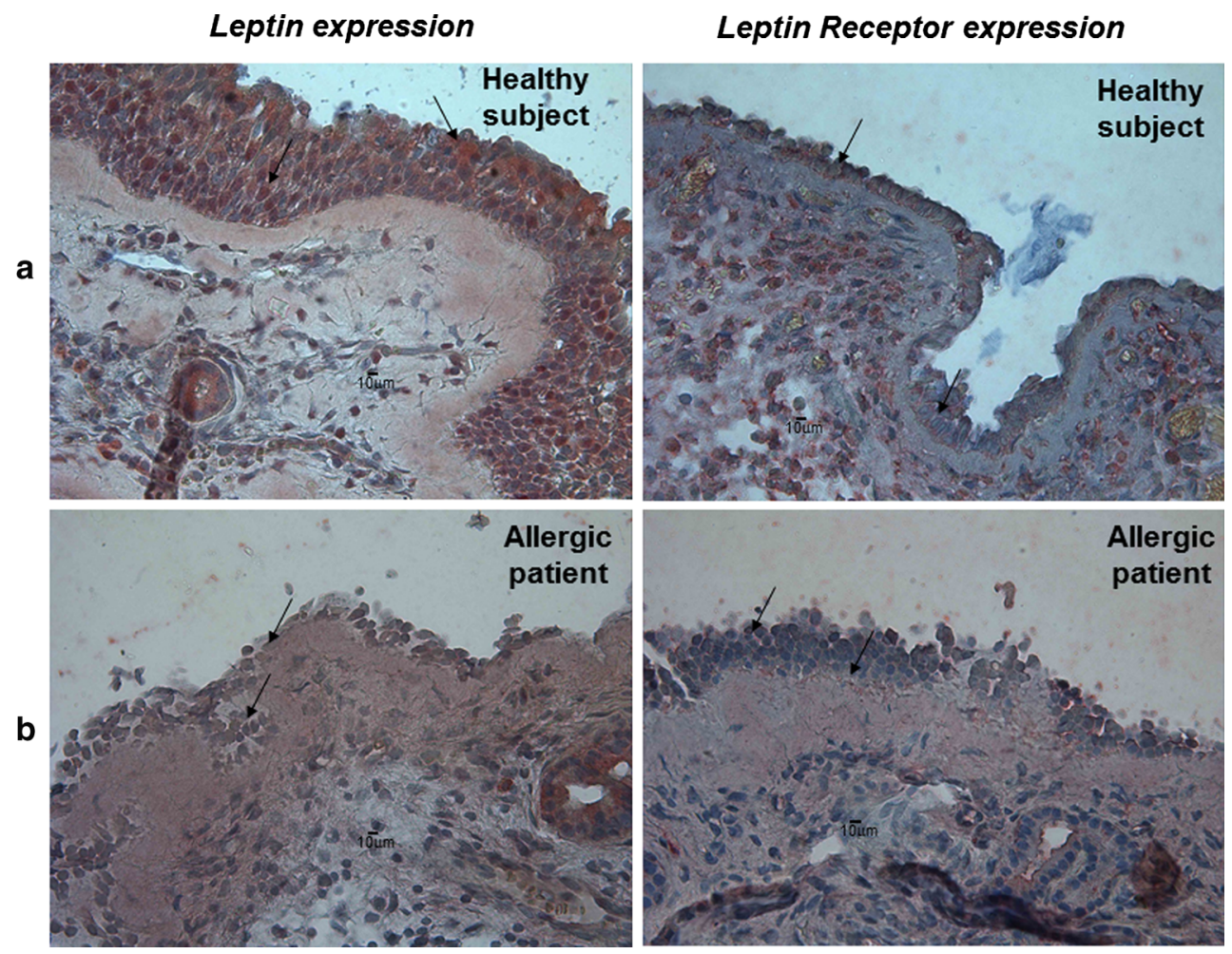

Fig. 2 Expression of leptin and leptin receptor in nasal biopsies. Inferior turbinate of nasal biopsies was collected from healthy not allergic control subjects $(n=3)$ and from allergic patients $(n=3)$. Leptin and leptin receptor were assessed by immunohistochemistry.

FF and with leptin alone $(p<0.03$ and $p<0.0002$, respectively), in comparison to medium alone (Fig. 3). On the other hand, significantly lower colony numbers were found in RPMI 2650 cells cultured with $\operatorname{rPar} j 1$ $(p<0.002)$ and with TGF- $\beta 1(p<0.03)$ alone in comparison to medium alone. The incubation with $\mathrm{FF}$ and $\mathrm{rPar}$ $\mathrm{j} 1$ and with FF and TGF- $\beta 1$ in combination was able to restore the cell proliferation and to significantly increase the colony numbers in comparison to rPar $\mathrm{j} 1(p<0.0002)$ or to TGF- $\beta 1(p<0.008)$ alone (Fig. 3). Leptin in combination with $\mathrm{rPar} \mathrm{j} 1 \mathrm{had}$ no effect on cell proliferation. Finally, the use of the pharmacological JAK2 inhibitor AG 490 was able to completely abolish the activity of leptin on cell proliferation $(p<0.0005)$, whereas it was able to counteract the proliferative effect of FF only when FF was present in combination with rPar j $1(p<0.02)$ suggesting that FF restores cell proliferation, reduced by rParj 1 exposure, by activating JAK2, a signal crucially involved in leptin/leptin receptor pathway (Fig. 3).

Effect of rPar j 1, FF, and cytokines on leptin and leptin receptor mRNA

To gain insight the mechanisms related to allergen exposure and corticosteroid treatment, we tested the effects of
Representative immunostaining in a healthy subject (a) and in a patient with allergic chronic rhinitis (b). The bars represent $10 \mu \mathrm{m}$, and the arrows indicates the epithelial cells. $\times 400$ magnification

rPar j 1, of FF, of leptin, and of TGF- $\beta 1$, alone and differently combined, on leptin and leptin receptor mRNA modulation. FF alone and in combination with leptin significantly increased the constitutive leptin receptor mRNA ( $p<0.001$ and $p<0.005$, respectively) (Fig. 4a). Furthermore, leptin, alone and in combination with $\mathrm{rPar} \mathrm{j} 1$, significantly increased the constitutive leptin receptor mRNA $(p<0.03$ and $p<0.001$, respectively). $\mathrm{rPar} \mathrm{j} 1$, TGF- $\beta 1$, and the association of FF and $\mathrm{rPar} \mathrm{j} 1$ had no effect on the modulation of the leptin receptor mRNA (Fig. 4a). All the tested stimuli were not able to modulate the leptin mRNA (Fig. 4b).

Effect of rPar J 1, FF, and cytokines on leptin/leptin receptor proteins

We next evaluated the ability of $\mathrm{rPar} \mathrm{j} 1, \mathrm{FF}$, recombinant human leptin and, TGF- $\beta 1$, alone or in combination, to modulate the expression of leptin and leptin receptor proteins in RPMI 2650 cells. rPar $\mathrm{j} 1$ and TGF- $\beta 1$ significantly decreased the leptin receptors expression in comparison to control ( $p<0.02$ and $p>0.04$, respectively). On the other hand, FF significantly increased constitutive leptin receptor expression $(p<0.02)$ and, when added to the cultures in combination to $\mathrm{rPar} \mathrm{j} 1$, was able to counteract its 


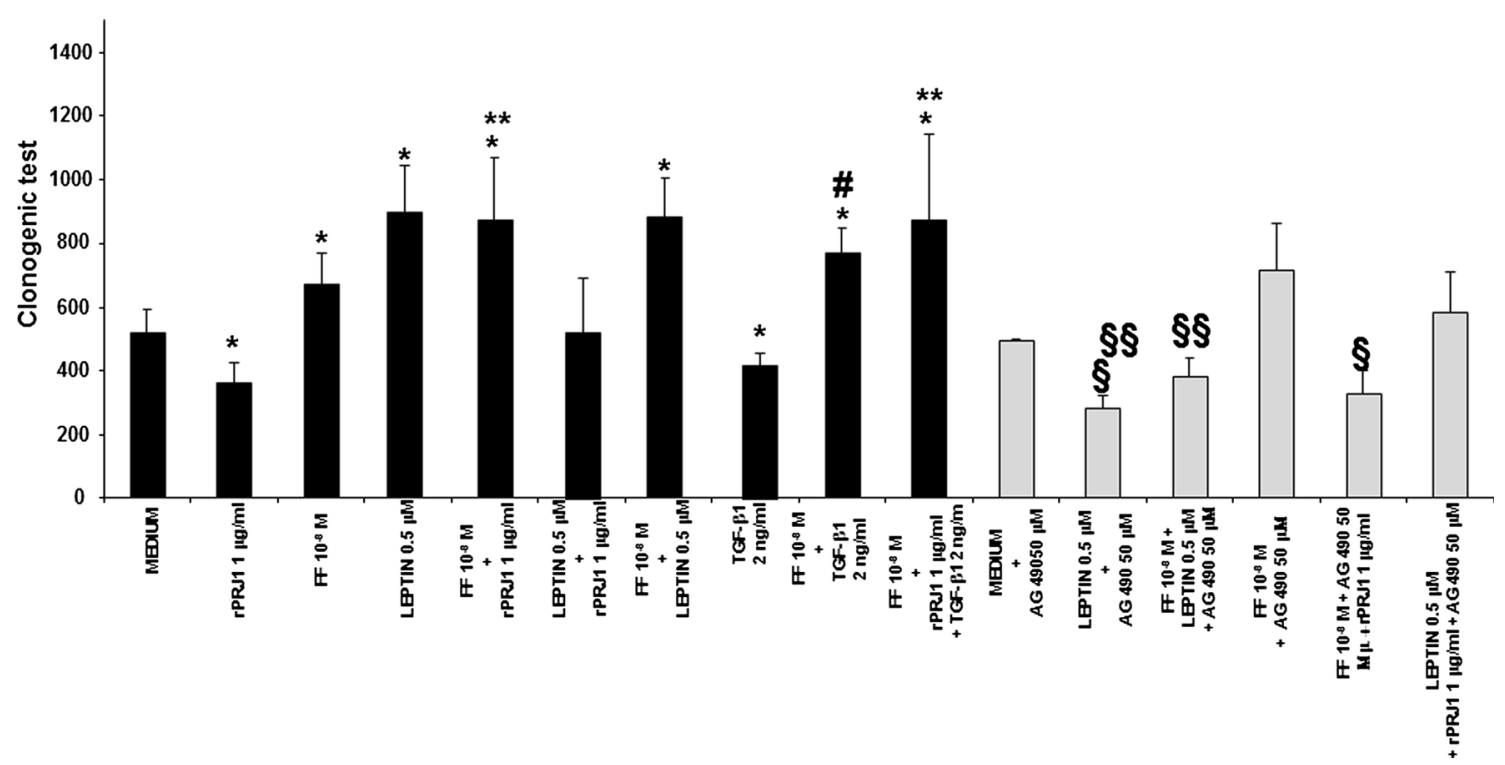

Fig. 3 rPar j 1, FF, and cytokines affect RPMI 2650 cell proliferation. This panel shows data (mean \pm SD) from five independent experiments. Gray histograms are with inhibitor AG 490. Significant difference * versus medium; ** versus rPar j 1 ; ${ }^{*}$ versus TGF $\beta 1 ; ~ \$$ versus medium + AG 490; ${ }^{\S \S}$ versus leptin. $p<0.05$, paired $t$ test
Fig. 4 rPar j 1, FF, and cytokines affect mRNA for leptin receptors. a This panel shows data for leptin receptors mRNA expression by real-time reverse transcription-PCR assays. GAPDH gene is used for normalization. Data are expressed as fold induction over baseline condition. Data (mean \pm SD) from three independent experiments. Significant difference $*$ versus medium. $p<0.05$, paired $t$ test. b This panel shows data for leptin mRNA expression by real-time reverse transcriptionPCR assays. GAPDH gene is used for normalization. Data are expressed as fold induction over baseline condition.

Representative data from two independent experiments
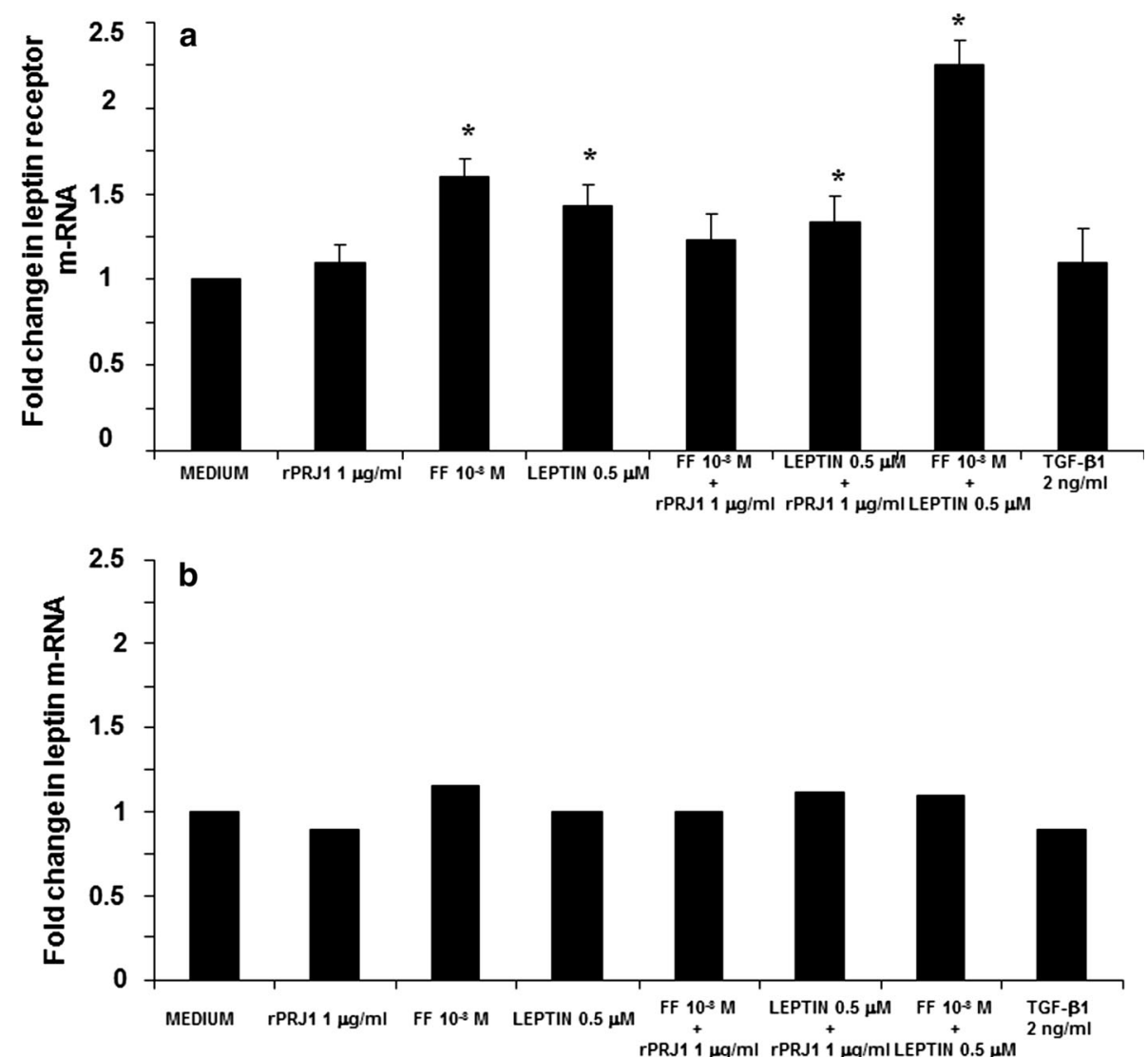

downregulatory effect $(p<0.03)$ (Fig. 5a, b). With regard to leptin protein expression, $\mathrm{rPar} \mathrm{j} 1$ significantly decreased $(p<0.003)$, whereas leptin and FF significantly increased
( $p<0.05$ and $p<0.03$, respectively) the expression of this protein in RPMI 2650 cells. When FF was added to the cultures in combination to rPar $\mathrm{j} 1$, it was able to counteract 
Fig. 5 rPar j 1, FF, and cytokines affect leptin receptor proteins in RPMI 2650 cells. a Physical parameters and overlay of fluorescence intensity (FL1) related to the expression of leptin receptor by flowcytometry analysis. b Data (mean $\pm \mathrm{SD}$ ) from eight independent experiments. Significant difference * versus medium; $* *$ versus $\mathrm{rPar} \mathrm{J} 1$. $p<0.05$, paired $t$ test
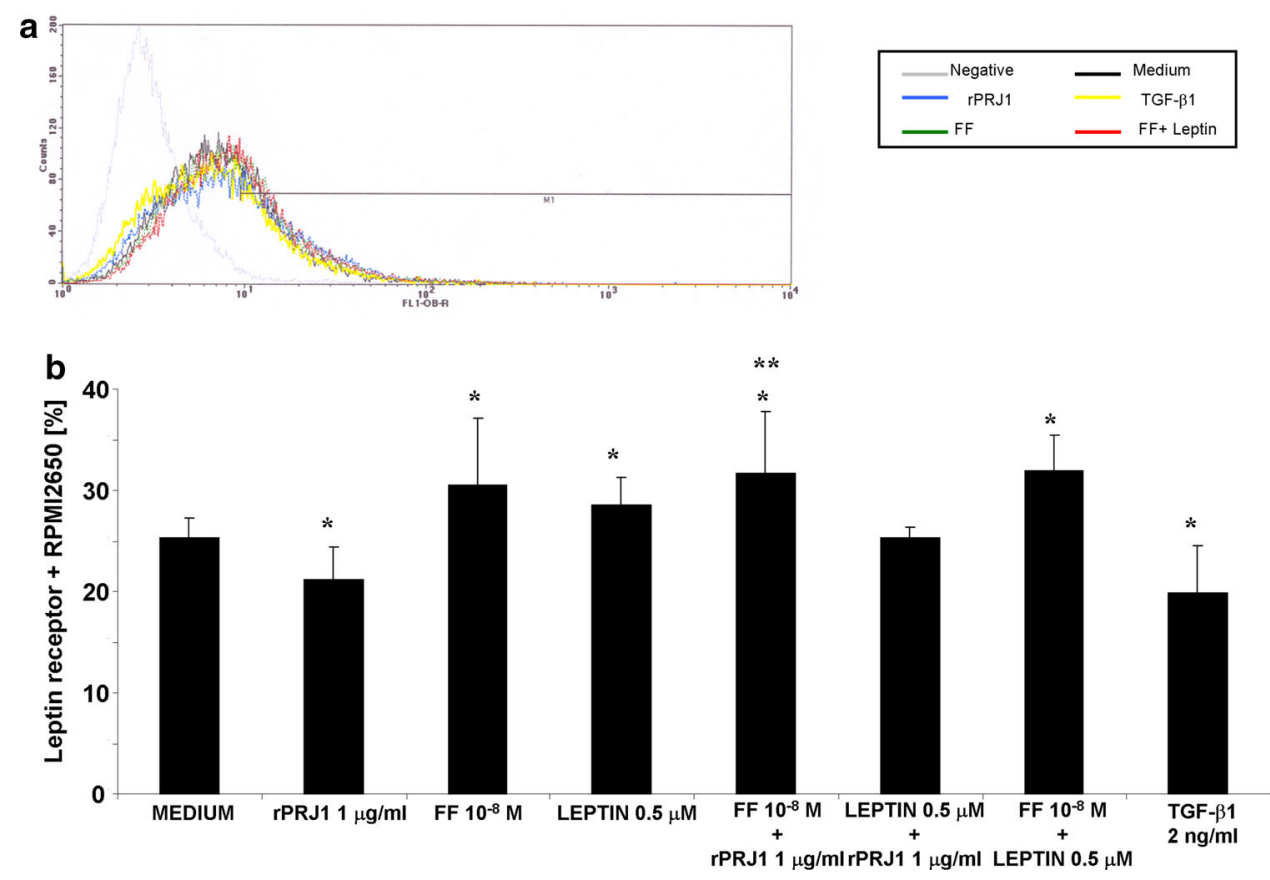

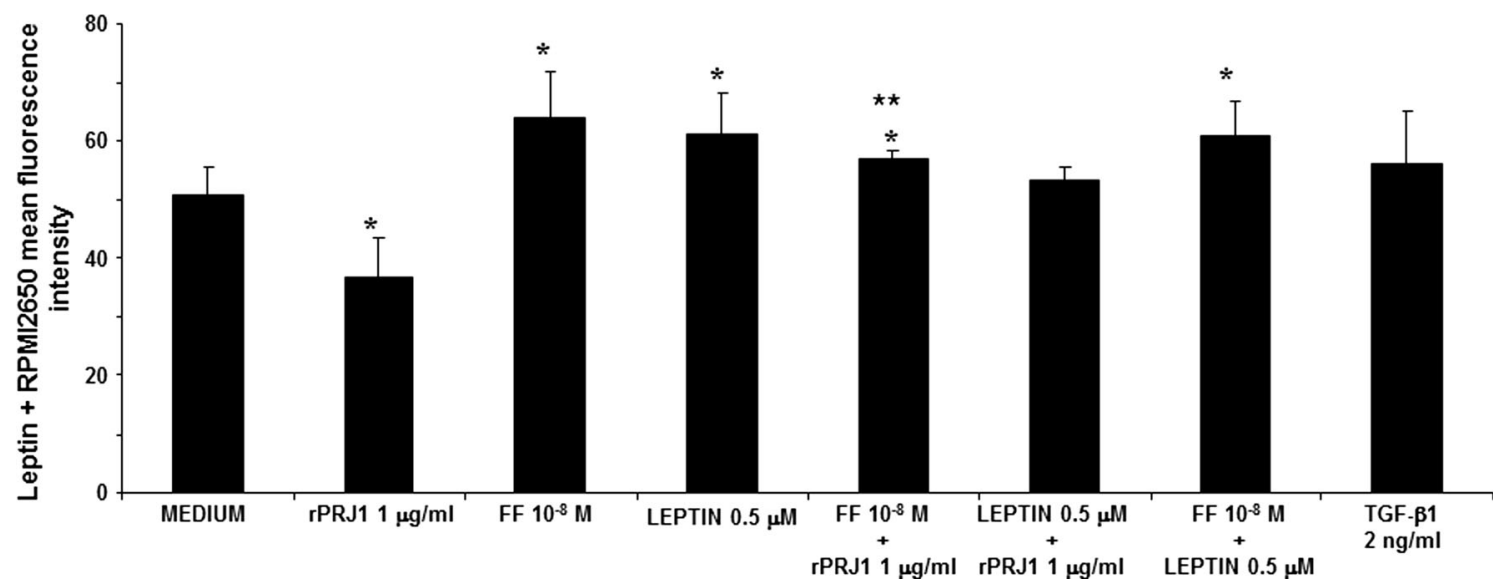

Fig. 6 rPar j 1, FF, and cytokines affect leptin protein in RPMI 2650 cells. This panel shows data (mean \pm SD) for leptin expression from eight independent experiments. Significant difference * versus medium; ** versus rPar J 1. $p<0.05$, paired $t$ test

the rPar $\mathrm{j} 1$ downregulatory effect $(p<0.007)$ (Fig. 6). No effect on leptin protein expression was exerted by TGF- $\beta 1$ and leptin in combination with rPar $\mathrm{j} 1$ (data not shown). The diluent of FF had no effect on both leptin and leptin receptors protein expression (data not shown).

Effect of rPar j 1, FF, and leptin on STAT-3 nuclear translocation

Since the activation of leptin/leptin receptor pathway leads to JAK2 activation and in turn to STAT-3 activation (3), the effects of rPar j 1, of FF, and of recombinant human leptin on STAT-3 nuclear translocation were evaluated.

After $24 \mathrm{~h}$ of incubation, rPar $\mathrm{j} 1$ significantly decreased the STAT-3 activation $(p<0.04)$. FF significantly increased the activation of STAT-3 $(p<0.02)$ and significantly counteracted $\mathrm{rPar}$ j 1 downregulatory effects $(p<0.004)$ (Fig. 7a). Leptin, at both 4 and $24 \mathrm{~h}$ of incubation, was not able to significantly counteract $\mathrm{rPar} \mathrm{j} 1$ effects (Fig. 7a). Leptin increased STAT-3 activation in a time-dependent manner reaching the maximum after $4 \mathrm{~h}$ of incubation $(p<0.02)$ and starting to decrease at $24 \mathrm{~h}$ (Fig. 7b).

\section{Discussion}

It has been widely shown that leptin is involved with different roles within the respiratory tract $[2,18]$, particularly with proliferative and protective roles in bronchial 
a

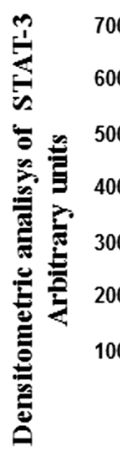

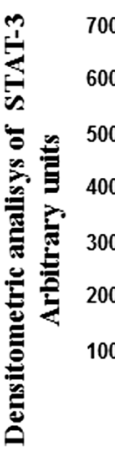

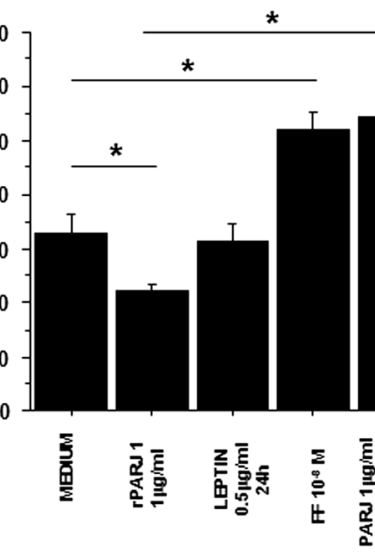

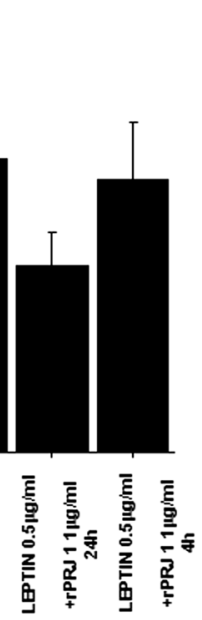

l=Medium
2=rPRJl;

2=rPRJl;

4=FF;
$4=\mathrm{FF}+\mathrm{PPRJ1}$

Cytoplasmatic Nuclear

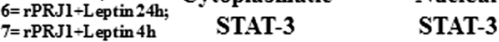

$\begin{array}{llllllllllllll}1 & 2 & 3 & 4 & 5 & 6 & 7 & 1 & 2 & 3 & 4 & 5 & 6 & 7\end{array}$

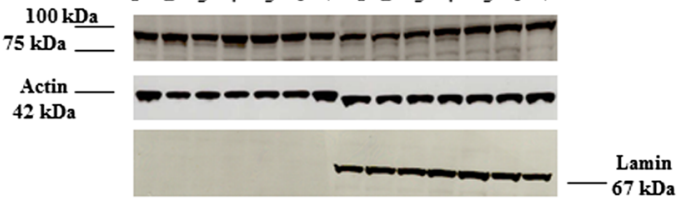

Fig. 7 rPar j 1, FF, and leptin affect STAT-3 nuclear translocation. STAT-3 nuclear translocation was assessed by western blot analysis on cytoplasmic and nuclear extracts, and the expression of STAT-3 was assessed using a monoclonal antibody anti-STAT-3. Membranes were then stripped and incubated with goat polyclonal anti- $\beta$-actin and with rabbit polyclonal anti-lamin B1. a The STAT-3 activation was assessed in cells cultured with $\mathrm{rPar} \mathrm{j} 1, \mathrm{FF}$, and leptin alone and in combination after $24 \mathrm{~h}$ of incubation. Signals corresponding to nuclear STAT-3 expression on the various western blots were semiquantified by densitometric scanning, normalized, and expressed

epithelial homeostasis [4, 6]. Allergen exposure is the first cause of AR, and topic corticosteroids treatment is the only efficient therapy to contrast its symptoms $[9,10]$. Presently, the literature lacks of in vitro evidences supporting the role of leptin in nasal epithelial homeostasis both in the presence and in the absence of allergens. In the present study, we demonstrate for the first time that FF contributes to maintain, in vitro, the nasal epithelium homeostasis in RPMI 2650 cells up-regulating leptin/leptin receptor pathway and increasing cell proliferation. The FF effects are dominant when compared to the allergen effects, since they persist also when the cells are cultured with FF combined with rPar $\mathrm{j}$.

The following new findings are reported: First, leptin and leptin receptor are expressed by RPMI 2650 cells (Fig. 1) and, in ex vivo conditions, by nasal epithelial cells (Fig. 2). Second, rPar j 1 and TGF- $\beta 1$ are able to significantly decrease cell proliferation (Fig. 3) other than to significantly decrease the leptin receptor expression both as mRNA (Fig. 4) and as protein expression (Fig. 5)

b

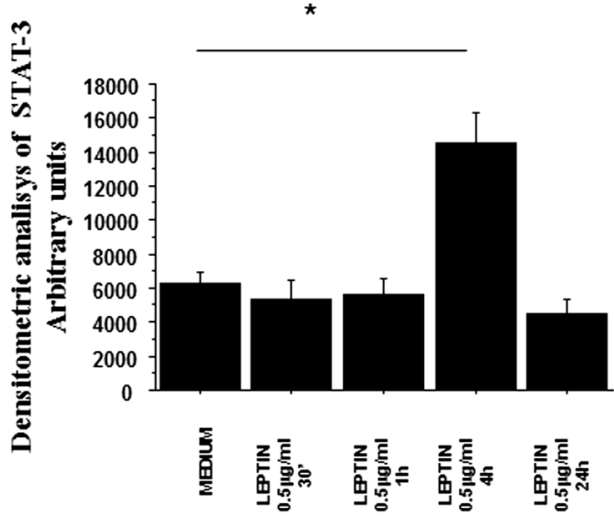

l=Medium;

$2=$ Leptin $30^{\circ}$

4=Leptin $4 \mathrm{~h} ; \quad$ Cytoplasmatic Nuclear

$5=$ Leptin 24

$100 \mathrm{kDa}$

$75 \mathrm{kDa}$

STAT-3

STAT-3

$\begin{array}{llllllllll}1 & 2 & 3 & 4 & 5 & 1 & 2 & 3 & 4 & 5\end{array}$

Actin
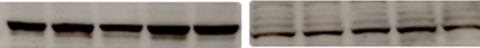

$42 \mathrm{kDa}$
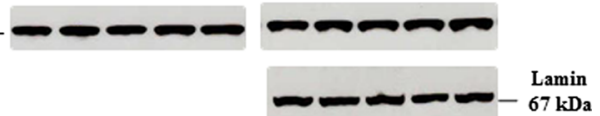

after correction with the density of the bands obtained for beta-actin and lamin B1. Data are expressed as arbitrary units \pm SD. $* p<0.05$. Representative western blot analyses from five independent experiments were shown. b Time course $(30 \mathrm{~min}, 1 \mathrm{~h}, 4 \mathrm{~h}$, and $24 \mathrm{~h}$ ) for leptin effects. Signals corresponding to nuclear STAT-3 expression on the various western blots were semiquantified by densitometric scanning, normalized, and expressed after correction with the density of the bands obtained for beta-actin and lamin B1. Representative western blot analyses from three independent experiments

expression. $\mathrm{rPar} \mathrm{j} 1$ is also able to significantly decrease leptin expression (Fig. 6). Third, FF significantly increases RPMI 2650 cell proliferation and the leptin/leptin receptors pathway expression, either in absence or in presence of rPar j 1 (Figs. 3, 5, 6). Fourth, leptin itself is able to significantly increase RPMI 2650 cell proliferation (Fig. 3) and the leptin/leptin receptor pathway expression (Figs. 4, 5,6 ), either in absence or in presence of FF. Pharmacological JAK2 inhibitor AG 490 completely abolishes leptin's activity on cell proliferation (Fig. 3). To our knowledge, this is the first in vitro study that evaluates the role of FF, alone or combined with rPar j 1 and with leptin, on cell proliferation and on the leptin/leptin receptors pathway expression.

The major allergen rParj 1 used in our experimental in vitro design is a specific isoform of 102 aminoacids (fully named Par j 1.0201). This isoform, despite has an almost undetectable endogenous endotoxin content ( $\leq 0.003 \mathrm{ng} \mathrm{LPS} / \mu \mathrm{g}$ of recombinant protein) [14] lacks, for its nature, of the aminoacidic tail that binds LPS. On the 
contrary, the longer isoform of the major allergen Parj 1 (fully named Par j 1.0101) is a specific isoform of 139 aminoacids containing also the LPS site binding [19]. It is well known that the total pollen extract is a mix of all allergens minor and major, but it is not standardized for each of major allergen [15] that contains all isoforms, included also the longer isoform of the major allergen Parj 1 , and than it is able to activate several intracellular signaling, including the leptin/leptin receptor pathway. These evidences further support the hypothesis that a purified major allergen, as Par j 1.0101 is able to induce, in an in vitro model, a negative modulation of the protective pathway of leptin and its receptor. Particularly, we observed that the major allergen rParj 1 used in our experimental in vitro design is able to significantly decrease leptin protein as well as its receptor expression. Here, recombinant human leptin is able to significantly increase both itself and its receptor protein expression. Interestingly, we found that the recombinant human fibrogenic cytokine TGF- $\beta 1$ modulates leptin/leptin receptor pathway reducing only leptin receptor expression. The role of TGF- $\beta 1$ is relevant in a long-term experimental condition (clonogenic test), since it exerts an anti-proliferative role, also by counteracting the exogenous recombinant human leptin and the FF proliferative roles. The positive regulation of cell proliferation is an event crucially involved in the repair processes also in nasal epithelium. In the nasal tract, upon injury, undifferentiated basal cells migrate from adjacent epithelium, spread over the denuded basement membrane, and proliferate in injured regions [7]. On the other hand, to better explain our results obtained by stimulating RPMI 2650 cells with FF, alone or in combination with either major allergen rParj 1 and with leptin itself, it has to be considered that intranasally administered corticosteroids have a wide margin of safety and are the mainstay of treatment for patients with moderate to severe AR, non-allergic rhinitis, and nasal polyposis [20]. The anti-inflammatory effects exerted by these drugs include the decrease of eosinophil and basophil cell infiltrates, of pro-inflammatory mediators, of vascular permeability, and of mucus production [21]. Corticosteroids can also exert immunomodulatory activities, since they can reduce the number of Th2 lymphocytes [22]. The local anti-inflammatory effects of corticosteroids play a relevant role in improving nasal epithelial histology. In this regard, it has been previously demonstrated that fluticasone propionate treatment for one year led to increased thickness of the nasal epithelium [13] and also yearlong therapy with FF increased the percentage of epithelium comprising intact columnar and ciliated epithelial cells [21]. In our study, only FF and leptin were able to modulate the expression of leptin receptor at both mRNA and protein levels. rPRJ 1 was able to modulate leptin receptor expression but not leptin receptor m-RNA. This discrepancy between leptin receptor mRNA and protein expression could be due to some post-transcriptional and/or post-translational processes. With regard to leptin expression, all the other tested stimuli were not able to modulate the m-RNA for leptin suggesting that the increased leptin protein expression is related to post-transcriptional regulatory events. Taken together, these findings suggest a potential homeostatic role of FF on the nasal epithelium, and the data from the present study demonstrate that FF exerts this homeostatic role by the activation of leptin/leptin receptor pathway in nasal epithelial cells. Leptin, in fact, has been shown to be a critical mediator of restitution following injury to the epithelium. In this regard, our previous study performed in vitro and ex vivo [6] concludes that firstly, in vitro the human bronchial epithelial cells express leptin/leptin receptors proteins, and fluticasone propionate increases leptin receptors expression; secondly, ex vivo in endobronchial biopsies from asthmatic patients of vary entities, the leptin/leptin receptors pathway is inversely correlated with features of remodeling as sub-epithelial thickness and TGF- $\beta 1$ expression, leading to the concept that the leptin/ leptin receptor pathway is involved in lung epithelial homeostasis in asthma, and its expression decreases in patients with uncontrolled and severe asthma. To reinforce this message, also another our previous study [5] performed ex vivo in endobronchial biopsies from COPD patients reports that in epithelial cells, the expression of leptin and its receptor is higher in healthy normal subjects than in smokers or in mild-to-severe COPD patients. Accordingly, in the present study, evaluating epithelial cells in inferior turbinate of nasal biopsies from three healthy not allergic control subjects and from three allergic patients, we found that there is a negative trend of the expression of leptin/leptin receptor pathway in the epithelium from allergic patients supporting the hypothesis of leptin as marker of epithelium homeostasis. On the other hand, leptin deficiency could lead to altered immune responses since this pathway affects both the innate and acquired immune systems. In this regard, it has been demonstrated that patients with reduced leptin levels have reduced CD4 T-cell populations (thus a reduced CD4/CD8 ratio), hyporesponsive $\mathrm{T}$-cells, severely reduced $\mathrm{IFN}-\gamma$ expression, and reduced levels of cytokines such as IL-10 and IL-4, while they have increased TGF- $\beta$ levels. Upon leptin replacement therapy, CD4 numbers, T-cell function, and IFN- $\gamma$ expression were all recovered, and TGF- $\beta$ levels dropped to normal levels [23]. Based on all these evidences, finally we performed further experiments, mainly directed to deeper investigate the signaling transduction of FF, leptin, and allergens in human nasal epithelial cells. The long isoform of the leptin receptor contains intracellular motifs required for the activation of multiple signaling 
including STAT-3. In this regard, it has been previously demonstrated that leptin increases cell proliferation in nucleus pulposus cells by up-regulating STAT- 3 activation [24]. We now demonstrate an activation of STAT-3 in leptin stimulated nasal epithelial cells after $4 \mathrm{~h}$ but not after $24 \mathrm{~h}$ of incubation suggesting that, upon STAT-3 activation, a negative feedback loop may be activated in nasal epithelial cells. Accordingly, leptin-induced signaling via STAT-3 rapidly activates the negative feedback regulator SOCS3, which inhibits leptin-induced signal transduction [25]. After $24 \mathrm{~h}$ of incubation, rPar J 1 reduces while FF increases STAT-3 activation and counteracts the effects of rPar J 1 on STAT-3 activation. Leptin alone (at 4 and at $24 \mathrm{~h}$ ) it was not able to revert the effect of rPar J 1 on STAT-3 activation. These findings suggest that at longer incubation time, FF might increase leptin/leptin receptor pathway effects also preventing the downregulation of STAT-3 activation due to allergen exposure.

\section{Conclusions}

In conclusion, the present study provides new evidences for leptin activity in the nasal tract, strongly supporting the concept that leptin could represent a key molecule for the epithelial homeostasis also in the upper airways. Furthermore, FF in the presence of the allergens insults is able to restore the leptin/leptin receptor pathway, supporting the concept that leptin is involved in human nasal epithelial homeostasis in AR.

Acknowledgments This work was supported by the Italian National Research Council and by GlaxoSmithKline.

\section{References}

1. Matarese G, Procaccini C, De Rosa V, Horvath TL, La Cava A (2010) Regulatory $\mathrm{T}$ cells in obesity: the leptin connection. Trends Mol Med. 16:247-256

2. Vernooy JH, Ubags ND, Brusselle GG, Tavernier J, Suratt BT, Joos GF, Wouters EF, Bracke KR (2013) Leptin as regulator of pulmonary immune responses: involvement in respiratory diseases. Pulm Pharmacol Ther 26:464-472

3. Mackey-Lawrence NM, Petri WA Jr (2012) Leptin and mucosal immunity. Mucosal Immunol 5:472-479

4. Bruno A, Siena L, Gerbino S, Ferraro M, Chanez P, Giammanco M, Gjomarkaj M, Pace E (2011) Apigenin upregulates leptin/ leptin receptor pathway and induces cell apoptosis in lung adenocarcinoma cell line. Eur J Cancer 47:2042-2051

5. Bruno A, Chanez P, Chiappara G, Siena L, Giammanco S, Gjomarkaj M, Bonsignore G, Bousquet J, Vignola AM (2005) Does leptin play a cytokine-like role within the airways of COPD patients? Eur Respir J 26:398-405

6. Bruno A, Pace E, Chanez P, Gras D, Vachier I, Chiappara G, La Guardia M, Gerbino S, Profita M, Gjomarkaj M (2009) Leptin and leptin receptor expression in asthma. J Allergy Clin Immunol 124:230-237 237.e1-4

7. Yan Y, Gordon WM, Wang DY (2013) Nasal epithelial repair and remodeling in physical injury, infection, and inflammatory diseases. Curr Opin Otolaryngol Head Neck Surg 21:263-270

8. Taildeman J, Demetter P, Rottiers I, Holtappels G, Bachert C, Cuvelier CA, Pérez-Novo CA (2010) Identification of the nasal mucosa as a new target for leptin action. Histopathology 56:789-798

9. Greiner AN, Hellings PW, Rotiroti G, Scadding GK (2011) Allergic rhinitis. Lancet 378:2112-2122

10. Brozek JL, Bousquet J, Baena-Cagnani CE, Bonini S, Canonica GW, Casale TB, van Wijk RG, Ohta K, Zuberbier T, Schünemann HJ, Global Allergy and Asthma European Network; Grading of Recommendations Assessment, Development and Evaluation Working Group [Collaborators (78)] (2010) Allergic Rhinitis and its Impact on Asthma (ARIA) guidelines: 2010 revision. J Allergy Clin Immunol 126:466-476

11. Salib RJ, Lau LC, Howarth PH (2005) The novel use of the human nasal epithelial cell line RPMI 2650 as an in vitro model to study the influence of allergens and cytokines on transforming growth factor-beta gene expression and protein release. Clin Exp Allergy 35:811-819

12. Pace E, Scafidi V, Di Bona D, Siena L, Chiappara G, Ferraro M, La Grutta S, Gallina S, Speciale R, Ballacchino A, Bachert C, Bousquet J, Gjomarkaj M (2012) Increased expression of IL-19 in the epithelium of patients with chronic rhinosinusitis and nasal polyps. Allergy 67:878-886

13. Baroody FM, Cheng CC, Moylan B, deTineo M, Haney L, Reed KD, Cook CK, Westlund RE, Sengupta E, Corey JP, Togias A, Naclerio RM (2001) Absence of nasal mucosal atrophy with fluticasone aqueous nasal spray. Arch Otolaryngol Head Neck Surg 127:193-199

14. Bonura A, Quaratino S, Gervasi F, Melis MR, Di Sano C, Colombo P (2013) Innate and adaptive immune responses to the major Parietaria allergen Par j 1 in healthy subjects. Immunobiology 218:995-1004

15. Colombo P, Bonura A (2006) Parietaria pollen allergens. Eur Ann Allergy Clin Immunol. 38:224-225

16. Pace E, Ferraro M, Mody CH et al (2008) Pleural mesothelial cells express both BLT2 and PPARa and mount an integrated response to pleural $\mathrm{LTB}_{4}$. J Immunol 181:7292-7299

17. Schmittgen TD, Livak KJ (2008) Analyzing real-time PCR data by the comparative C(T) method. Nat Protoc 3:1101-1108

18. Malli F, Papaioannou AI, Gourgoulianis KI, Daniil Z (2010) The role of leptin in the respiratory system: an overview. Respir Res 11:152. doi:10.1186/1465-9921-11-152

19. Bonura A, Corinti S, Schiavi E, Giacomazza D, Gianguzza F, Di Felice G, Colombo P (2013) The major allergen of the Parietaria pollen contains an LPS-binding region with immuno-modulatory activity. Allergy 68:297-303

20. Klossek JM, Laliberté F, Laliberté MF, Mounedji N, Bousquet J (2001) Local safety of intranasal triamcinolone acetonide: clinical and histological aspects of nasal mucosa in the long-term treatment of perennial allergic rhinitis. Rhinology. 39:17-22

21. Fokkens WJ, Rinia B, van Drunen CM, Hellings PW, Hens G, Jansen A, Blom H, Wu W, Clements DS, Lee LA, Philpot EE (2012) No mucosal atrophy and reduced inflammatory cells: active-controlled trial with yearlong fluticasone furoate nasal spray. Am J Rhinol Allergy. 26:36-44

22. Malmhäll C, Bossios A, Pullerits T, Lötvall J (2007) Effects of pollen and nasal glucocorticoid on FOXP3+, GATA-3+ and T-bet + cells in allergic rhinitis. Allergy 62:1007-1013

23. Farooqi IS, Matarese G, Lord GM, Keogh JM, Lawrence E, Agwu C, Sanna V, Jebb SA, Perna F, Fontana S et al (2002) Beneficial effects of leptin on obesity, T cell hyporesponsiveness, 
and neuroendocrine/metabolic dysfunction of human congenital leptin deficiency. J Clin Invest. 110:1093-1103

24. Li Z, Shen J, Wu WK, Yu X, Liang J, Qiu G, Liu J (2012) Leptin induces cyclin D1 expression and proliferation of human nucleus pulposus cells via JAK/STAT, PI3K/Akt and MEK/ERK pathways. PLoS One 7(12):e53176
25. Wunderlich CM, Hövelmeyer N, Wunderlich FT (2013) Mechanisms of chronic JAK-STAT3-SOCS3 signaling in obesity. JAK-STAT 2013(2):e23878 